\title{
LA NASCITA DELLE RISERVE DI LIBRI ANTICHI IN ITALIA
}

\section{Andrea De Pasquale}

La nascita delle riserve di libri rari a stampa nella biblioteche europee trova tradizionalmente origine nell'opera compiuta da Joseph Basile Bernard Van Praet alla Bibliothèque Nationale di Parigi, quando, assunto nel 1784 dopo essere stato collaboratore del libraio Guillaume De Bure, provvide alla ricognizione dei libri confiscati ai conventi, individuando nel 1794 le categorie di libri rari e meritevoli di essere conservati in luoghi distinti e protetti (le réserves) rispetto alle collezioni generali ${ }^{1}$.

L'introduzione di tale pratica in Italia è poco conosciuta ed è mancata a tutt'oggi un'indagine complessiva che permetta di fare luce sui tempi e i modi in cui i bibliotecari, di fronte alle collezioni, si siano attivati a definire il concetto di raro, ad individuare i materiali con specifiche caratteristiche e a stabilire modalità e classificazioni per salvaguardarli in luoghi separati.

Molto limitati sono pure i riferimenti nella manualistica biblioteconomica. Leopoldo Della Santa, nel suo trattato del $1816^{2}$, diceva a tal proposito cose generiche: "Prossime alle Stanze dei Bibliotecari ho collocate quattro grandi Librerie, che io chiamo Librerie scelte, per dover queste servire a più preziosi, e rari Volumi: cioè alle Edizioni del primo secolo, a quelle di Lusso, ai Manoscritti, ed alle Stampe. Dette Librerie le ho situate prossime ai Bibliotecari per maggior loro comodità, e facilità

1 Dominique VARry, Joseph Van Praet, in Histoire des bibliothèques françaises, vol III, Les Bibliothèques de la Révolution et du XIX esiècle, a cura di D. VARry, Paris, Promodis-Cercle de la librairie, 1991, pp. 302-303. Le categorie comprendevano i libri su pergamena, gli incunaboli, le importanti edizioni dei secoli seguenti, con particolare riguardo alle prime, i grand papiers, i libri illustrati, le opere degli stampatori celebri, i libri annotati e le legature di pregio.

2 Leopoldo Della SAnta, Della costruzione e del regolamento di una pubblica universale biblioteca con la pianta dimostrativa. Trattato, Firenze, presso Gaspero Ricci da S. Trinita, 1816, p. 34. 
alla vigilanza che sopra di esse devono avere; dovendosi a loro soltanto indirizzare quelli ai quali bisogni, o che sieno curiosi di vedere quanto di più prezioso la Biblioteca possiede: delle quali Librerie terranno solo essi le chiavi, e ne saranno i loro particolari Custodi".

Più dettagliato era invece Agostino Salvioni che nel 1845 parlava dell'esistenza di sezioni per incunaboli ed edizioni di tipografi celebri' "Le edizioni del secolo XV., volgarmente del quattrocento, cotanto pregievoli perché le prime di ogni autore, dette principes, tengono luogo di codici manoscritti, avranno posto in separati scaffali, seguendo l'ordine cronologico. É costumanza usata in alcune librerie di collocare separatamente le edizioni di celebri tipografi, quali sono gli Aldi, gli Elzeviri, i Comini, ed altri di simile celebrità. Ma ora questa specie di tipomania, per buone ragioni, va scemando alquanto di moda. Vengono per ultimo i codici manoscritti. Dilicati, e preziosi cimelii son questi di ogni pubblica, e privata biblioteca. Saranno essi gelosamente custoditi in appositi armadj, che dai latini, con tecnica voce detti furono plutei. Il Bibliotecario non ne permetterà la ispezione, che con sorveglianza diligentissima".

Si è quindi cercato di analizzare le storie delle biblioteche governative italiane, eredi di quelle degli antichi Stati preunitari, al fine di verificare quando e come è emersa questa procedura, con particolare riguardo all'arco cronologico tra XVIII e XIX secolo ${ }^{4}$.

Torino, Biblioteca dell'Università (ora Biblioteca Nazionale UNIVERSITARIA)

Il più antico caso noto di creazione di riserve per libri antichi è quello della Biblioteca dell'Università di Torino, fondata nel 1723 dal re Vittorio Amedeo II di Savoia. Il bibliotecario abate Francesco Domenico Bencini, entrato in servizio nel 1729 , mentre procedette al riordino e alla Bergamo, dalla Tipografia Crescini, 1845, pp. 24-25.

4 Sul tema: David Mскіттекіск, The invention of rare books. Private Interest and Public Memory, 1600-1840, Cambridge, Cambridge University Press, 2018. 
catalogazione dei fondi, costituì, all'interno degli armadi già destinati a conservare i manoscritti ${ }^{5}$, degli spazi specifici dove andare ad accogliere pure gli incunaboli, ammontanti a quell'epoca a ben 250 pezzi (armadio M), i libri manoscritti e stampati di romanzi, di figure, di disegni e altri libri (rari) "che devono custodirsi sotto chiave" (armadio G) e i libri musicali ${ }^{6}$. Si assiste così per la prima volta alla definizione di un embrionale concetto di rarità che comprendeva, accanto ai manoscritti, i libri del XV secolo, quelli illustrati e quelli con testo musicale, a cui si aggiungevano evidentemente altre tipologie di libri a stampa non meglio definiti, ritenuti meritevoli di essere conservati sotto chiave ${ }^{7}$.

Mentre le altre classificazioni andarono successivamente abolite, la sezione degli incunaboli venne potenziata e arricchita con le acquisizioni delle biblioteche confiscate dei conventi in eta napoleonica (1803). A quell'epoca il vicebibliotecario e bibliografo barone Giuseppe Vernazza di Freney (1808-1814) provvide a rivedere l'intero impianto classificatorio degli incunaboli (e i post-incunaboli degli inizi del XVI

Archivio di Stato di Torino, Corte, Regi Archivi, cat. 9, mazzo 1, n. 1: Indice de' libri manoscritti ebraici, greci, latini, italiani e francesi i quali la R.M. del re di Sardegna ha tolti dal suo Regio Archivio per rendere riguardevole la Biblioteca della sua Regia Università di Torino ed ha ordinato sian depositati nella camera alla Biblioteca medesima vicina. I medesimi si sono ordinati in armarj e con chiave custoditi e nell'istessi armarj distribuiti $i$ primi secondo le lingue ebrea, e greca. Il restante secondo le classi, e materie, che trattano. Da Francesco Domenico Bencini ab. Commendatario perpetuo di S. Ponzio di Nizza, e Prefetto dell'Archivio e Biblioteca della Regia Vniversità.

6 Archivio di Stato di Torino, Corte, Regi Archivi, cat. IX, mazzo 1, n. 1: Indice de' libri stampati rinserrati nell'armario notato con la lettera $\mathrm{M}$ dell'Archivio de' libri manoscritti della Bibl. della Regia Vniversità di Torino. Sono i libri posti con ordine cronologico tipografico i quali sono stati stampati dal principio della stampa sino a tutto l'anno 1500. Titoli dei fascicoli interni: Indice de' libri posti nell'armario contrassegnato con la lettera $G$ [...] Situato nell'archivio de MSS. che stanno nella Biblioteca della Regia Vniversità di Torino nel quale si contengono i libri MSS. e stamp. di romanzi, di figure stampate di dissegni \& altri libri, che devono custodirsi sotto chiave; Musicae libri cum notis impressis, et MSS. extantes cum reliquis olim libris inter MSS. codices

$7 \quad$ Andrea De Pasquale, Il sapere per tutti. La politica bibliotecaria a Torino tra XVII e XIX secolo, Savigliano, L'artistica, 2006, pp. 32-34. 
secolo), organizzandoli, per formato, dal più grande al più piccolo, in una nuova sezione che prese il nome di XV (per il secolo XV a cui appartengono la maggior parte dei pezzi $)^{8}$.

Nelle stessi anni nella medesima Biblioteca si assiste anche alla costituzione di una specifica sezione dedicata ai libri a stampa ebraici denominata Hebr., dove vennero fatti confluire i libri in tale lingua provenienti dalla sezione soprattutto di biblica dell'antica collezione dei Savoia e i libri dell'abate Tommaso Valperga di Caluso, celebre ebraista, che li donò alla Biblioteca sia in vita nel 1809 sia per testamento nel 1815, e di cui fu fatto un catalogo dal suo discepolo, l'abate Amedeo Peyron?'

Sempre negli anni successivi alla Restaurazione, venne anche costituita una sezione di edizioni aldine. Una parte di queste apparteneva ai nuclei antichi della Biblioteca, tra cui quello della casa reale sabauda, ma esso venne incrementato con donazioni e acquisti negli anni successivi, tanto che nel 1852 il bibliotecario Bernardino Peyron citava l'esistenza di un fondo specifico, di cui curò la redazione del catalogo che andò distrutto

Andrea De Pasquale, Vere fenici. Gli incunaboli della Biblioteca Nazionale Universitaria di Torino, Cinisello Balsamo, Silvana editoriale, 2014. pp. 3-13.

9 Andrea De Pasquale, Le edizioni ebraiche della Biblioteca Nazionale Universitaria di Torino, in Judaica Paedemontana. Libri e argenti da collezioni piemontesi, Cinisello Balsamo, Silvana Editoriale, 2015, pp. 29-35; Id., Le collezioni di libri a stampa ebraici della Biblioteca Nazionale Universitaria di Torino e della Biblioteca Palatina di Parma, in Il collezionismo di libri ebraici tra XVII e XIX secolo. Atti del convegno, Torino, 27 marzo 2015, a cura di Chiara Pilocane e Amedeo Spagnoletto, Supplemento al vol. 82, n. 2-3 (maggio-dicembre 201) de "La Rassegna mensile di Israel", Firenze, Giuntina, 2017, pp. 23-31. 
verosimilmente nell'incendio del 1904, impedendo oggi di comprenderne la reale consistenza ${ }^{10}$.

\section{Milano, Biblioteca di Brera (ora Biblioteca Nazionale Braidense)} Il direttore Luigi Lamberti, noto grecista e bibliofilo, in carica dal 1803, affidò al sottobibliotecario Morali il compito di scorporare le edizioni di pregio dalle collezioni generali, con particolare riguardo alle edizione dei Manuzio.

Il Lamberti fece raccogliere le edizioni aldine appartenenti ai nuclei costitutivi della Biblioteca (i fondi del conte Carlo Pertusati e del cardinale Angelo Maria Durini), e quelli dei conventi soppressi (tra cui il fondo dei Gesuiti che conteneva una sessantina di edizioni soprattutto di Paolo e di Aldo il giovane), e ne acquistò parecchie altre (circa 150 probabilmente), con una prevalenza di quelle stampate da Aldo il vecchio. La ricerca di edizioni aldine proseguì anche negli anni successivi, incentivata dalla disponibilità sul mercato antiquario di pezzi a prezzi accessibili, in quando ancora non oggetto di bibliofilia, come avverrà in seguito.

La catalogazione del fondo fu cominciata sulla base dei lavori di Renouard, che aveva visitato la Biblioteca proprio durante la direzione di Lamberti, comprendendo non solo le edizioni di Aldo il vecchio, ma anche quelle di tutti gli eredi, classificate per anno ${ }^{11}$. Lamberti ne affidò la schedatura, insieme a quella degli incunaboli, all'abate Robustiano

10 Andrea De Pasquale, Aldo e gli altri nelle raccolte della Biblioteca Nazionale Universitaria di Torino, in Hypnerotomachia Polophili, ubi humana omnia non nisi somnium esse docet, atque obiter plurima scitu sane quam digna commemorat, Venetiis, in cedibus Aldi Manutii, accuratissime mense decembri 1499. Testi critici, in allegato alla ristampa facsimilare, Savigliano, L'Artistica editrice, 2014, pp. 9-22; Id., Il collezionismo di aldine nelle biblioteche dell'Italia nord-occidentale del XIX secolo: i casi delle biblioteche nazionali di Milano e Torino in Five centuries later. Aldus Manutius: culture, typography and philology, a cura di Natale VAcalebre, Firenze, L.S. Olschki; Milano, Biblioteca Ambrosiana, 2018, pp. 181-190.

11 Il primo catalogo, ancora conservato in Biblioteca, è: Catalogus senioris Aldi editionum quas extant in Brajdensi bibliotheca relié avec Catalogus editionum Aldinarum in Braydensi Bibliotheca asservantur. Turritatus, et Aldi filii.. 
Gironi, vicebibliotecario e anche lui grecista, il quale diventò direttore dal 1813, alla morte di Lamberti, fino al 1838.

Quest'ultimo progettò un nuovo catalogo della Biblioteca, procedendo alla divisione per classi e schedando, oltre alle aldine, anche gli incunaboli e le edizioni rare, che furono sistemate in una nuova sala a lato di quella del catalogo, detta "l'aula della Sala Nuovissima", costruita nel 1811, e volle testimoniare la sua passione per la storia del libro e per gli studi bibliografici facendo rappresentare, tra gli uomini illustri, nella volta della sala cataloghi, Aldo Manuzio, accanto al presunto prototipografo milanese Filippo Lavagna ${ }^{12}$. Pochi anni dopo, nel 1840, le edizioni aldine furono radunate nella Sala dei manoscritti e rari, andando a formare il fondo di aldine, procedendo contestualmente al cambiamento delle relative segnature ${ }^{13}$.

Venezia, Biblioteca di S. Marco (ora Biblioteca Nazionale Marciana)

Meno definita è la situazione di Venezia, dove sicuramente il prefetto Jacopo Morelli, attivo in Marciana per ben quarant'anni (1778-1819), al momento delle soppressioni conventuali, individuò, all'interno delle biblioteche, gli incunaboli ${ }^{14}$. Nuove collocazioni, con la separazione dei pezzi più rari, vennero create in occasione del trasferimento della Biblioteca Marciana a Palazzo Ducale nel 181l, collocazioni che vennero poi nuovamente stravolte quando essa rientrò nella sua sede originaria al Palazzo delle Procuratie nel 1924.

12 Andrea De Pasquale, L'histoire du livre dans le décor des bibliothèques d'Italie au XIX siècle, in Bibliothèques. Décors (XVII-XIX siècle), a cura di Frédéric Barbier, István Monok, Andrea De Pasquale, Paris, Éditions des Cendres ; Budapest, Biblioteca dell'Accademia Ungherese scientifica ; Roma, Biblioteca nazionale centrale, 2016, pp. 249-264.

13 Andrea De Pasquale, Il collezionismo di aldine nelle biblioteche dell'Italia nordoccidentale del XIX secolo, cit.

14 Marino Zorzi, La Libreria di San Marco : libri, lettori, societa nella Venezia dei Dogi, Milano, Mondadori, 1987, pp. 357-364. 
In una statistica delle biblioteche governative del 1893 si segnalava che alla Marciana erano presenti collezioni di aldine (raccolta "quasi completa"), giuntine e cominiane ${ }^{15}$. Non sappiamo quando vennero costituite, ma la loro origine va attribuita con tutta probabilità all'opera di Morelli.

\section{Parma, Biblioteca Palatina}

La presenza di collocazioni distinte per i cimeli della biblioteca è attestata fin dal momento della fondazione e si deve all'opera del suo fondatore, il padre Paolo Maria Paciaudi, direttore dal 1761 al 1774.

Sappiamo infatti che i manoscritti e gli incunaboli (detti "quattrocentisti") erano tenuti separati dal corpo principale della Biblioteca, il grande salone detto Galleria Petitot, e sistemati nell'ufficio del Paciaudi, che fungeva anche da luogo di conservazione e forse anche di consultazione, allocati in banconi in stile identico a quelli della Galleria ${ }^{16}$. Gli incunaboli mantennero quindi una collocazione separata e, al momento dell'acquisizione della biblioteca dei duchi di Parma ${ }^{17}$, vennero distinti in Incunaboli Parmensi (Inc. Parm.) e Incunaboli Palatini (Inc. Pal.).

Successivamente l'interesse della Biblioteca si spostò verso il collezionismo di edizioni bodoniane. Angelo Pezzana, direttore della Parmense dal 1814 al 1862, trascorse anni a ricostruire la totalità degli annali bodoniani ricercando sul mercato antiquario anche esemplari

Ministero della Pubblica Istruzione, Notizie storiche, bibliografiche e statistiche sulle biblioteche governative del Regno d'Italia, Roma, Tipografia elzeviriana di Adelaide ved. Pateras, 1893, p. 115.

16 Sulla presenza di questi materiali nella stanza cfr. Ireneo Afrò, Il Parmigiano servitor di piazza ovvero dialoghi di Frombola ne' quali dopo varie notizie interessanti su le pitture di Parma si porge il catalogo delle principali, Parma, Carmignani, 1794, p. 56. Cfr. anche Andrea De PAsquale, Parma città d'Europa. Le memorie del padre Paciaudi,per la Biblioteca Parmense, Parma, Museo Bodoniano, 2008; Il Ducato in scena, catalogo della mostra, a cura di Andrea De Pasquale e Giovanni Godi, Parma, STEP, 2009.

17 Libri a corte. Le biblioteche dei Duchi di Parma conservate nella Biblioteca Palatina, a cura di Andrea De Pasquale, Parma, MUP, 2011. 
stampati su supporti speciali come la pergamena e la seta, che incluse in una specifica sezione, strettamente connessa con il restante materiale bodoniano $^{18}$.

Pezzana si interessò anche ad altri libri su supporti speciali, quali pergamena e carta colorata, o stampati in poche copie o in esemplari unici. Nel 1842 egli acquistò la biblioteca del bibliografo Bartolomeo $\mathrm{Gamba}^{19}$, ricca di edizioni rarissime, specialmente del XVIII e XIX secolo, stampate in limitate copie perché spesso non destinate alla vendita, sovente d'occasione, su pergamena e su carte colorate. Delle edizioni su queste ultime fece redigere pure uno specifico catalogo ${ }^{20}$.

Per tali libri, distinti per materiale e rientranti nella categoria delle curiosità bibliografiche, Pezzana costituì apposite sezioni, insieme ad un'altra specifica destinata ad accogliere i libri più rari e preziosi scorporati dalle collezioni generali ${ }^{21}$.

Egli inoltre riuscì ad acquistare nel 1816 la raccolta di manoscritti e di edizioni ebraiche e orientali dell'abate Gian Bernardo De Rossi, celebre ebraista e docente di letteratura orientale. Si trattava di una collezione già specifica dedicata ai libri in alfabeto ebraico, che mantenne nella sua forma, distinguendo soltanto i materiali tra manoscritti e stampati ${ }^{22}$.

18 Andrea De Pasquale, Le edizioni bodoniane su pergamena, "Crisopoli. Bolletino del Museo Bodoniano di Parma”, 14 (2011, ma 2013), n.s. II, pp. 83-106.

19 Andrea De Pasquale, Collezioni scelte. Rarità bibliografiche acquisite nel XIX dalla Biblioteca Palatina di Parma, Parma, MUP, 2010.

20 Biblioteca, Archivio storico, Cat. 94/9: Libri stampati sopra carte colorate o distinte. Il catalogo è redatto presumibilmente da Giovanni Mantelli, conservatore delle stampe, negli anni di direzione del Pezzana.

21 Collezioni scelte. Rarità bibliografiche acquisite nel XIX dalla Biblioteca Palatina di Parma, a cura di Andrea De Pasquale, Parma, MUP, 2010.

22 Andrea De Pasquale, Exoticis linguis. Libri ebraici e orientali della Biblioteca Palatina di Parma, Parma, MUP, 2009; Id., Le collezioni di libri a stampa ebraici della Biblioteca Nazionale Universitaria di Torino e della Biblioteca Palatina di Parma, cit., pp. 31-36. 
Modena, Biblioteca Estense

La collezione separata di incunaboli venne fondata nel 1760 dal bibliotecario Francesco Antonio Zaccaria, in margine a quella dei manoscritti, e di essa venne redatto dallo stesso Zaccaria un catalogo ${ }^{23}$.

La statistica del 1893 dice che erano possedute anche collezioni di edizioni di tipografi celebri: di aldine (circa 600), di giuntine (più di 500), e "molte degli Stefani, dei Griffi, dei Torrentini, dei Marcolini, dei Bodoni, nonché molte plantiniane, elzeviriane, cominiane", ma non è noto a che epoca vennero costituite, probabilmente nel corso del XIX secolo, perdendo poi la loro fisionomia separata ${ }^{24}$.

Firenze, Biblioteca Magliabechiana e Biblioteca Palatina (ora Biblioteca Nazionale Centrale di Firenze)

Situazioni analoghe si riscontrano pure a Firenze.

Un'attenzione specifica alle rarità bibliografiche inizia ad apparire quando nel 1771 Pietro Leopoldo decise di riunire la Biblioteca Mediceo Palatina Lotaringia alla Biblioteca Magliabechiana. Vista la necessità di liberare in fretta il palazzo Pitti, il bibliotecario Targioni ripropose il vecchio ordinamento presso la biblioteca Magliabechiana, che era stata ampliata di cinque stanze destina ai "libri regi", vicino alle raccolte dei manoscritti e dei libri a stampa di pregio, e, con l'aiuto del sottobibliotecario Ferdinando Fossi, cominciò a esaminare il fondo per scegliere i duplicati, con l'accortezza di conservare gli esemplari di pregio per edizioni, legatura o decorazione.

Nel 1818 il granduca Ferdinando III di Toscana promosse l'acquisizione, presso gli eredi, della collezione del bibliofilo Gaetano Poggiali che comprendeva ben 440 codici e 480 edizioni del XV secolo, oltre alle serie degli autori italiani, dei novellieri e dei libri di poesia e belle arti.

\footnotetext{
23 Domenico FAva, Catalogo degli incunaboli della R. Biblioteca Estense di Modena, Firenze, Leo S. Olschki, 1928, p. 1.

24 Ministero della Pubblica Istruzione, Notizie storiche, bibliografiche e statistiche sulle biblioteche governative del Regno d'Italia, cit., p. 177.
} 
Contestualmente si pose attenzione proprio alle edizioni del XV secolo e di lì a poco si compilò un catalogo specifico, dedicato a quelle italiane e intitolato "Catalogo delle edizioni di opere italiane eseguite nel secolo XV". In esso compare una specifica segnatura per gli incunaboli contraddistinta dall'indicazione "Ed. del XV secolo" seguita da un numero progressivo da 1 a 416. Tale catalogo non contiene solo le edizioni già di proprietà del Poggiali, ma anche acquisti successivi (è documentata un'acquisizione del 1826), ma pare certo che la sua compilazione sia contestuale all'acquisto della biblioteca Poggiali e che esso fu usato fino a quando la Biblioteca Palatina venne fusa con la Magliabechiana. Oltre a catalogare in esso la raccolta Poggiali, a partire da una certa data, successiva al 1828, si iniziò a registrare anche gli incunaboli già conservati in Biblioteca, ma collocati in altre sezioni ${ }^{25}$.

Successivamente all'interno della Biblioteca Palatina si creò una classe specifica per "Edizioni del secolo XV", chiamata ED. XV, la prima della serie, come dimostra il Catalogo della Biblioteca privata di Sua Altezza Imperiale e Reale il Granduca di Toscana" che attesta il posseduto della Biblioteca fino a tutto il febbraio $1827^{26}$.

Del tutto specifica e particolare è invece una collezione con apposita sezione speciale che formò il granduca Ferdinando III e che, insieme alla sezione dei classici greci e latini, curò personalmente con spirito bibliofilico. Si tratta della raccolta delle edizioni degli Elzevier, iniziata dal suddetto granduca, e incrementata successivamente con due acquisti specifici: il 19 ottobre 1838 con 70 edizioni; e nel 1843 con 163 pezzi comprati da Giuseppe Molini per conto della Biblioteca palatina dall'asta promossa dal libraio Motteley. Il nucleo di libri delle Repubbliche pare invece provenire dagli acquisiti dei doppi della

25 Marielisa Rossi, Bibliofilia, bibliografia e biblioteconomia alla corte dei granduchi di Toscana Ferdinando III e Leopoldo II. Itinerarî esplorativi fra cataloghi e documenti della Biblioteca Palatina lorenese, Manziana (Roma), Vecchiarelli editore, 1996, pp. 108-113.

26 Marielisa Rossı, Bibliofilia, bibliografia e biblioteconomia, cit., p. 156. 
biblioteca di lord Spencer a loro volta provenienti dall'acquisto della biblioteca di Reviczki venduta allo Spencer nel $1790^{27}$.

Nell'inventario di consegna della Biblioteca Palatina, redatto dal Molini nel 1827, il fondo ammontava a ben 600 pezzi $^{28}$. Le edizioni recano la segnatura Elz. e costituivano una sezione specifica come si evince dal Catalogo della Biblioteca privata già citato del 1827 in cui compare la classe "Elzeviri" denominata ELZEV.

\section{Roma, Biblioteca Casanatense}

Il padre Masettí, storico della Biblioteca, racconta che le edizioni del $\mathrm{XV}$ secolo che si trovavano in origine disperse nel grande salone della Biblioteca insieme alla totalità del patrimonio posseduto, vennero radunate e concentrate nella prima stanza precedente il Salone e affacciata sulla chiesa di S. Ignazio a cura del padre Giacomo Magno, prefetto dal 1798 al 1840.

La stanza prese il nome di "Camera dei Quattrocentisti" e il Magno catalogò, tra il 1800 e il 1820, le edizioni in un repertorio di tre volumi che segue l'ordine cronologico ${ }^{29}$.

\section{Napoli, Biblioteca Nazionale}

Le origini della Biblioteca Nazionale di Napoli sono tradizionalmente datate all'ultimo ventennio del secolo XVIII quando venne dato ordine, nel 1780, di trasportare al palazzo degli Studi la biblioteca dei Farnese, a sua volta condotta a Napoli da Parma da re Carlo III di Borbone. A tale biblioteca si aggiunsero anche la Biblioteca Palatina, che si conservava a Capodimonte e i libri della soppressa congregazione dei Gesuiti espulsi

27 Marielisa Rossi, Bibliofilia, bibliografia e biblioteconomia, pp. 120; 128-129.

28 Della raccolta è pure disponibile un catalogo (Edizioni degli stampatori Elzeviere Le "Repubbliche" stampate dagli Elzevier) e ne esisteva pure un altro manoscritto su pergamena intitolato Catalogus editionum Elzeviriorum ora perduto.

29 Marina Panetta, Rita Ajello, Il fondo incunaboli e rari, in La Biblioteca Casanatense, a cura di Angela Adriana Cavarra, Firenze, Nardini, 1993, p.124. 
nel 1767. Il tutto al fine di aprire una biblioteca pubblica, in aggiunta a quella di Sant'Angelo al Nilo che era estremamente ridotta ${ }^{30}$.

I bibliotecari Andrea Belli, entrato come aiutante nel 1784 e poi bibliotecario dal 1788 al 1817, e il celebre grecista Pasquale Baffi, intrapresero la catalogazione degli stampati concludendo i lavori nel 1794; quindi passarono alle edizioni aldine, agli incunaboli, agli atlanti e ai volumi con figure. Progettarono anche di sistemare in un'unica stanza tutti gli incunaboli acquisiti fino al 1794 aggiungendovi anche successive edizioni a stampa fino al 1520 (ma lasciandone ancora molte nella sala principale) e ivi collocando pure le aldine provenienti quasi totalmente dalle raccolte farnesiane. Contestualmente, nel 1788, i bibliotecari proposero l'emanazione di un provvedimento che vietasse l'esportazione dai confini di manoscritti e libri del XV secolo e che spronasse i Presidi delle Province dove erano rimaste delle biblioteche gesuitiche a far trasportare alla biblioteca reale i cataloghi al fine di provvedere alla scelta di libri, con particolare riguardo agli "antichi manoscritti ed edizioni notoriamente del XV secolo".

Era attivo in Biblioteca, aperta poi solo il 13 gennaio 1804, anche Lorenzo Giustiniani, già vicebibliotecario al momento dell'apertura della biblioteca e poi retrocesso, insigne bibliografo, autore di un Saggio storicocritico sulla tipografia del Regno di Napoli del 1793 che ebbe una successiva edizione dal titolo Saggio storico-critico sulla tipografia del Regno di Napoli nel secolo XV che non poté essere pubblicata per la morte dell'autore. Egli, incaricato di studiare delle sezione specifiche dei manoscritti durante la direzione del prefetto Juan Andres (in carica dal 1806), propose anche una serie di migliorie per Biblioteca che prevedevano la suddivisione dei libri in 35 classi e la costituzione di collezioni speciali degli Aldi e degli Stefani, la collocazione a parte delle edizioni napoletane e il rifacimento del catalogo degli incunaboli.

Nel 1822 la Biblioteca venne in possesso di una cospicua parte della raccolta del marchese Francesco Antonio Taccone, venduta al re 
Gioacchino Murat e già collocata nel Monastero di Monteoliveto e che alla Restaurazione aveva avuto l'indicazione di essere divisa tra la Reale e l'Universitaria. Per effettuare la cernita venne fatta una classificazione del materiale in quattro gruppi che comprendevano "Libri rari e pregevoli che mancano alla Real Biblioteca Borbonica", libri già possedutí, ma "rari e pregevoli " che "sarebbe più decoroso che ve ne siano doppii esemplari" e quindi "le collezioni degli Aldi, dei Baskerville, dei Barbou, de' Didot, de' Bodoni, de' Due Ponti, degli Stefani, degli Elzeviri, de' Grifi, de' Torrentino, de' Gioliti, de' Giunti, de' Comini" ${ }^{31}$.

Nello stesso 1822 il Giustinani e soprattutto il p. Francesco De Lecteriis provvidero alla scelta delle edizioni più rare del secolo XV disponendole in una sezione a parte distinta in 27 plutei, individuarono le edizioni stampate a Napoli e in altre città del Regno, collocandole in 4 plutei, separarono le Aldine, le edizione degli Stefani e dei Giunti e le Bodoniane; inoltre crearono una collezione di libri di somma rarità usciti dai torchi dei migliori tipografi di ogni nazione e di ogni tempo. Il lavoro dovette essere concluso nel 1824 e il De Licteriis pubblicò il volume Codicum saeculo XV impressorum qui in Regia Bibliotheca Borbonica adservantur Catalogus, Napoli, ex Regia Typo., 1828-1833 con Supplementum del 1841.

La revisione dell'inventario fu ordinata il 1 marzo 1853 a mons. Rossi e Giovanni D’Avossa che produssero nel 1854 un nuovo strumento. Da esso si evince che gli incunaboli erano conservati negli scaffali IXIII, XIX-XX, e che nei primi plutei di questi vi erano anche edizioni posteriori. Inoltre lo scaffale XIV conteneva la collezione Bodoniana; il XV le collezione degli Stefani, dei Giunta, degli Elzeviri; il XVI collezioni di opere rare in edizioni diverse, posteriori al 1500; gli scaffali XVII e XVIII le Aldine $e^{32}$.

31 Guerriera Guerrieri, La Biblioteca nazionale «Vittorio Emanuele III» di Napoli, cit., pp. 20-21

32 Guerriera Gubrrieri, La Biblioteca nazionale «Vittorio Emanuele III» di Napoli, pp. 138-139 


\section{Altre biblioteche}

Con tutta probabilità situazioni analoghe a quelle riportate erano diffuse in altre biblioteche. L'unica fonte a disposizione è la già citata indagine condotta nel 1893 sulle biblioteche governative pubblicata per il congresso internazionale dei bibliotecari tenutosi a Chicago in quell'anno, da cui si evince che praticamente tutte le biblioteche avevano sezioni dedicate ai libri più rari ${ }^{33}$.

Per l'Universitaria di Bologna si parla di una raccolta di edizioni aldine "raccolte togliendole dalle diverse donazioni fatte in diverse epoche e dai diversi acquisti fatti dal Senato".

Alla Pubblica di Lucca è citata la raccolta di 417 edizioni citate nella Crusca, mentre all'Universitaria di Napoli di "una pregevole raccolta di edizioni aldine in $n$. di 150 volumi".

Per l'Universitaria di Messina si ricordava "una collezione di 447 opere rare, più o meno pregiate dai bibliografi, e di edizioni aldine. Essa è costituita da libri scelti nell'antica suppellettile e da quelli che man mano si vanno trovando nelle librerie claustrali a questa biblioteca devolute. Fra i libri di questa collezione ve n'ha qualcuno, di cui non conoscesi altro esemplare".

Nell'Universitaria di Sassari invece si affermava che "vanno considerate le edizioni Aldine, dei Giunti, dei Sermartelli, dei Bladi, dei Giolito, del Valgrisi, dei Grifi, dei Roville, degli Stefani, dei Badio, dei Colines, degli Elzeviri delle quali la Biblioteca possiede una discreta collezione".

\section{Conclusioni}

Dall'analisi effettuata si può sicuramente affermare che in Italia assistiamo già nella prima metà del XVIII secolo alla costituzione di collezioni distinte di edizioni rare, per ragioni di salvaguardia del materiale e di tutela dai furti.

33 Ministero della Pubblica Istruzione, Notizie storiche, bibliografiche e statistiche sulle biblioteche governative del Regno d'Italia, cit., rispettivamente, pp. 227, 160, $275,261,331$. 
Se la pratica di enucleare i manoscritti era diffusa fin dalle origini, l'esempio più precoce di costituzione di una collocazione separata per incunaboli pare quello della Biblioteca universitaria di Torino risalente al 1729-1730, in linea anche con la diffusione degli interessi per la stampa delle origini. Successivamente con la disponibilità dei primi repertori, già a partire dalla seconda metà del XVIII secolo, tali nuclei si ingrandirono soprattutto all'epoca delle soppressioni conventuali quando le biblioteche degli ordini religiosi vennero acquisite da biblioteche pubbliche e riordinate, scremate dei doppi e analizzate individuando in esse i pezzi più rari. Abbiamo quindi collezioni distinte di incunaboli a Modena (1760), Parma (circa 1761), Napoli (1794), Milano (1811), Venezia (circa 1811), Firenze (1818) e Roma in Biblioteca Casanatense (1820).

Diffuse sono anche le raccolte di edizioni Aldine (Aldo il vecchio, Paolo, Aldo il giovane, Andrea Torresano), nate sulla scorta in prima battuta del repertorio di Burgassi ${ }^{34}$, ma poi soprattutto degli Annales di Renouard sia nella prima edizione del 1803 , sia sopratutto nel supplemento del $1812^{35}$. Sono infatti note a Napoli (1794 e circa 1806), Milano (1811), Venezia (circa 1811), Torino (circa 1820).

Attestate sono pure collezioni specifiche di libri ebraici, nate però sostanzialmente da raccolte private confluite in Biblioteca, quali quelle di Torino (1815 circa) e Parma (1816).

A partire dagli anni '20 del XIX secolo si iniziano anche collezionare raccolte dedicate a specifici tipografi celebri, strettamente connesse

34 Antonio Cesare Burgassi, Serie dell'edizioni aldine per ordine cronologico e alfabetico, Padova, 1790 e quindi ristampato nel 1803: cfr. la ristampa Serie dell'edizioni aldine per ordine cronologico ed alfabetico (Firenze, 1803), a cura di Piero Scapecchi, Bologna, A. Forni, 2013.

35 Antoine-Augustin Renouard, Annales de l'imprimerie des Alde, ou l'histoire des trois Manuce et de leurs éditions, A Paris, chez A.-A. Renouard, 1812; 1825²; $1834^{3}$. 
con la pubblicazione di repertori, annali e studi ${ }^{36}$, tra cui spiccano gli Étiennes, gli Elzevirs, e Bodoni. Sono i casi delle Biblioteche di Napoli (1822), di Firenze (circa 1827) e ovviamente di Parma (circa 1840).

In questa ultima città negli stessi anni si formano anche collezioni di libri stampati su supporti speciali (seta, pergamena, carta colorata) e in copie limitate o uniche.

La pratica di costituire sezioni distinte per raccolte di libri a stampa rari e di pregio, andò avanti nel secolo e trovò la sua ultima definitiva applicazione a Roma alla Biblioteca Nazionale Vittorio Emanuele, con il direttore reggente Carlo Castellani, studioso di storia del libro e di incunaboli, che fin dal 1875 iniziò la costituzione di sezioni specifiche

36 Per gli Elzevier: Auguste Joseph de Reume, Recherches historiques, généalogiques et bibliographiques sur les Elsevier, (Ad. Wahlen et compagnie, Bruxelles, 1847, 124 p. ); Charles Pieters, Annales de l'Imprimerie Elsevirienne et histoire de la famille des Elsevier et de ses editions, (C. Annoot-Braeckman, Gand, 1851; seconde édition, revue et augmentée: C. Annoot-Braeckman, Gand, 1858). Per gli Estienne: Antoine-Augustin Renouard, Annales de l'Imprimerie des Estienne, ou Histoire de la famill e des Estienne et de leurs éditions, Paris, Renouard, 1843. Per la Tipografia Volpi-Cominiana: Fortunato Federici, Annali della tipografi VolpiCominiana, 1809. Per i Giunta: M. BANdinI, De Florentina Iuntarum typographia eiusque censoribus, Lucae 1791, pp. 1-19; Antoine-Augustin Renouard, Notice sur les Junte et la liste sommaire de leurs éditions jusqu'en 1550, in Annales de l'imprimerie des Alde, ou Histoire des trois Manuce et de leurs éditions, III, Paris 1825, pp. 321-409. Per Francesco Marcolini, editore di Aretino e del celebre libro delle Sorti: Gaetano ZACCARIA, Catalogo ragionato di opere stampate per Francesco Marcolini da Forlì, con memorie biografiche del medesimo tipografo raccolte dall'avv. Raffaele De Minicis, Fermo, Tip. de' Fratelli Ciferri, 1850; Scipione Casali, Annali della tipografia veneziana di Francesco Marcolini da Forlì, Forlì, presso Matteo Casali, 1861. Per Lorenzo Torrentino: Domenico Moreni, Annali della Tipografia Fiorentina di Lorenzo Torrentino Impressore Ducale. Edizione seconda, corretta, e aumentata, 2a ed., Firenze, 1819. (prima edizione: Firenze 1811. Per Bodoni: Giuseppe De Lama, Vita dl cavaliere Giambattista Bodoni tipografo italiano, Parma, Stamperia Ducale, 1816, con il secondo volume che comprende gli annali delle sue edizioni. 
dedicate ai tipografi illustri (Giunta, Blado, Paganino, Torrentino, Stefani, Elzeviri, Bodoni, Aldo) all'interno dei fondi della Biblioteca ${ }^{37}$.

La testimonianza più evidente di questo interesse è rappresentata dall'ideazione del ciclo pittorico della volta del salone del piano terra della Biblioteca, che venne ornata, oltre che dalle raffigurazioni degli stemmi delle principali città italiane e della Sicilia, e da medaglioni con putti rappresentati le arti, e, nelle lunette a lato delle finestre, da marche di antichi tipografi ${ }^{38}$.

Anche il conte Domenico Gnoli, successivo direttore a partire dal $1881^{39}$, formò, "accanto al corpo generale della biblioteca", "certe librerie speciali, o collezioni tenute a parte sia per il pregio loro, sia perché oggetto di curiosità, sia per altre ragioni". Giuseppe Fumagalli, suo antico collaboratore e poi direttore della Biblioteca Braidense ed estensore di un importante manuale sulla collocazione dei libri, ricordava infatti l'esistenza nella biblioteca romana de "la collezione di Incunabuli, e di altre specialità tipografiche (edizioni Aldine, Elzeviriane, Bodoniane ecc.), i Rariores, i volumi postillati, le legature pregevoli, le opere riccamente illustrate; le librerie cinese e giapponese; le Res Societatis

37 Cfr. i Registri relativi alle collezioni delle edizioni dei Giunta, di Blado ed eredi, di Paganino de' Paganini, di Torrentino; degli Stefani, degli Elzeviri e di Bodoni, rilegati insieme, a cui è premessa una nota di mano di Castellani che illustra che la ricognizione delle edizioni dei tipografi celebri iniziò già nel 1875 e venne proseguita poi dall'assistente Severini. Non venne invece realizzato un catalogo delle aldine in quanto si annotarono le segnature in corrispondenza delle descrizioni del citato repertorio di Burgassi.

38 Si possono riconoscere infatti quelle di Christophe Plantin, Henri Estienne, Giovanni Andrea Valvassori, Johann Froben, i Manuzio, Vincenzo Valgrisi, Antonio Blado, Giovanni Antonio Farri, l'Accademia della Crusca, i Giunta, Giorgio Marescotti, Bartolomeo Sermartelli, Gabriele Giolito de' Ferrari, Giorgio Varisco, Simon de Colines (Paris) e Sébastien Gryphe. Cfr. Andrea De Pasquale, L'histoire du livre dans le décor des bibliothèques d'Italie au XIX siècle, in Bibliothèques. Decorations, cit., pp. 249-264.

39 Andrea De Pasquale, La politica bibliotecaria di Domenico Gnoli per la Vittorio Emanuele, in Al regno di Romolo succede il regno di Numa. Domenico Gnoli direttore della Biblioteca nazionale centrale di Roma, a cura di A. De Pasquale, Roma, Biblioteca nazionale centrale, 2017, pp. 32-33. 
Jesu; la raccolta topografica romana; la raccolta bibliografica, e due librerie di consultazione; e finalmente la splendida biblioteca storica del Risorgimento italiano" 40 .

A queste raccolte si deve aggiungere anche quella dedicata allo stampatore Antonio Blado e ai suoi eredi, iniziata da Castellani e proseguita da Gnoli, che promosse la realizzazione del catalogo, a cura di Giuseppe Fumagalli e Giacomo Belli ${ }^{41}$.

Lo stesso Fumagalli, nel suo citato manuale Della collocazione dei libri nelle pubbliche biblioteche ${ }^{42}$, codificava per la prima volta in Italia il concetto di "Riserva", dove "Le opere più preziose che la biblioteca possiede, devono, per ogni giusta cautela, conservarsi in sede separata, in scaffali chiusi con reti o con vetri, e serrati a chiave"43, e ne elencava

40 Giuseppe Fumagalui, Della collocazione dei libri nelle pubbliche biblioteche, Firenze, Sansoni, 1890 (rist. anast, con introduzione di Giovanni Di Domenico), Manziana, Vecchiarelli, 1999), pp. 95-96.

41 Giuseppe Fumagalli - Gioacchino Belli, Catalogo delle edizioni romane di Antonio Blado asolano ed eredi (1516-1593) possedute dalla Biblioteca Nazionale Centrale Vittorio Emanuele di Roma. Con una introduzione storico-bibliografica, Firenze-Roma, tip. Bencini, Ministero della Pubblica Istruzione, 1891. Giuseppe Fumagalli pubblicò anche una monografia dedicata a Blado (Giuseppe Fumagalli, Antonio Blado tipografo romano del secolo XVI. Memoria storico-bibliografica, Milano, Hoepli, 1894).

42 Giuseppe Fumagalli, Della collocazione, cit., p. 96.

43 Secondo Fumagalli, Della collocazione, cit., pp. 33-34, la riserva avrebbe conservato non solo volumi, ma anche opuscoli preziosi, per i quali si suggeriva di dare ad ognuno "una separata legatura in modo da poterlo collocare a parte senza necessità di chiuderlo in una busta di miscellanee". Anzi sottolineava che in presenza di miscellanee, nel caso di legature non di pregio, si sarebbero dovuti sciogliere e legare ciascun opuscolo separatamente. Questo ai fini della tutela, ritenendo molto pericoloso dare in lettura opuscoli rarissimi rilegati. Fumagalli consigliava anche di estrapolare delle collezioni generali i volumi di pregio senza cambiante segnatura, sostituendoli con una tavoletta che ne segnalava il trasferimento e dando alla serie un solo numero progressivo. Inoltre sottolineava il fatto che la chiave degli scaffali della riserva non dovevano essere nella mani dei distributori ma conservate o dal capoufficio o da altro impiegato di rango superiore di sua fiducia e sotto la sua responsabilità. 
le categorie ricomprese, corrispondenti a specifici interessi bibliofilici, e in particolare:
A. Edizioni xilografiche;
B. Incunabuli, e particolarmente i paleotipi anteriori al 1470;
C. Libri condannati e perseguitati, o soppressi dall'autore medesimo;
D. Libri fattisi rari per altre ragioni, sia perché logorati e dispersi dal continuo e insipiente uso (come gli antichi trattati di abaco, esemplari calligrafici, Donati pro puerilis, edizioni di letteratura popolare, romanzi cavallereschi ecc.) sia perché incendi od altre eccezionali circostanze ne distruggessero la maggior parte degli esemplari;
E. Libri stampati privatamente od a ristrettissimo numero di copie;
F. Libri incisi, e preziosi per grande valore artistico;
G. Libri stampati su pergamena, su seta o su altre materie singolari;
H. Esemplari preziosi per la loro provenienza, per autografi di insigni personaggi ecc.;

I. Legature storiche ed artistiche

Successivamente lo stesso Fumagalli, nella nuova edizione che fece nel 1916 del manuale Hoepli sulla Bibliografia di Giuseppe Ottino, riprendeva il tema del collezionismo di libri rari, soffermandosi sul concetto di rarità e giungendo a distinguere tre classi di libri rari e pregevoli, in particolare quelli per il contenuto, quelli per l'edizioni e quelli per le particolarità degli esemplari ${ }^{44}$. Tutte le tre classi erano suddivise ancora in 25 categorie, in particolare:

44 Giuseppe Fumagalli, La Bibliografia, terza edizione interamente rifatta e ampliata del Manuale di Bibliografia di Giuseppe Ottino, Milano, Hoepli, 1916, ripresa poi nella quarta edizione, riveduta e arricchita da Giuseppe Fumagalli e Olga Pinto, del 1935, alle pp. 347-399. 
1. Libri in lingue orientali o in altre lingue poco conosciute e poco parlate

2. Libri figurati

3. Le storie locali

4. Opere antiche di geografia, antiche relazioni di viaggi, antiche carte geografiche e marine e atlanti

5. Opere antiche di matematica, di meccanica, di gnomica, di astronomia, di astrologia, prognostici, opere di alchimia di medicina, chirurgia, ostetricia e farmacia, erbari e ricettari, specialmente se con figure

6. Opere di musica antica e di storia del teatro

7. Vecchi giornali e almanacchi

8. Libri di letteratura popolare, canzonette, giuochi, raccolte d'indovinelli, riboboli e proverbi, novellette, leggende sacre, storie amorose e cavalleresche, romanzi di cavalleria, teatro popolare, vecchi testi dialettali.

9. Libri su argomenti curiosi

10. Facezie, satire, libri a chiave

11. Libri erotici

12. Libri condannati e perseguitati

13. Libri espurgati in esemplari risparmiati dalla censura, libri con carticini

14. Curiosità letterarie minori

15. Opere da biblioteca (grandi corpi storici e letterari, le grandi opere d'arte, di storia naturale, di viaggi, le grandi collezioni di riviste, di atti accademici, ecc.)

16. Edizioni del sec. XV

17. Edizioni originali

18. Edizioni di celebri tipografi, come gli Aldi, i Giunta, i Giolito, gli Stefani, gli Elzeviri, i Comini, i Bodoni

19. Edizioni di stamperie private o particolari

20. Edizioni tirate a piccolissimo numero di esemplari, quasi sempre fuori commercio 
21. Libri divenuti rari per singolari circostanze (incendi, naufragi, ecc.) che ne hanno distrutto il maggior numero delle copie o perché furono soppressi dagli autori stessi

22. Curiosità tipografiche, per esempio i libri stampati in colori

23. Esemplari stampati su pergamena, o su altre carte speciali. Esemplari miniati. Esemplari speciali, arricchiti dei disegni originali delle vignette o delle tavole fuori testo, o con prove di incisioni in diversi stati, prove avanti lettera, prove d'artista (cioè in tirature speciali), saggi, prove in colori, ecc.

24. Esemplari postillati

25. Esemplari con legature artistiche o storiche o stemmate.

Nonostante però Fumagalli avesse riproposto il collezionismo dei libri rari, nelle biblioteche pubbliche la moda era ormai tramontata. Esse infatti avevano smesso di comprarne, se non in casi eccezionali e si era ormai abbandonato l'interesse per la materialità del libro; inoltre le antiche collocazioni per tipografi erano state chiuse, preferendo concentrare nei magazzini riservati i libri di pregio per formato e non più per categorie, come accadde anche alla Biblioteca nazionale di Roma. 
LES BIBLIOTHÈQUES ET L'ÉCONOMIE DES CONNAISSANCES Bibliotheken und die ÖKonomie des Wissens

$$
145^{\circ}-185^{\circ}
$$

Colloque international - Internationale Tagung 9-13 avril/April 2019 Sárospatak (Hongrie/Ungarn)

Édité par

Frédéric Barbier, István Monok et Andrea Seidler 


\section{L'Europe en réseaux}

Contribution à l'histoire de la culture écrite 1650-1918

Vernetztes Europa

Beiträge zur Kulturgeschichte des Buchwesens 1650-1918

Édité par

Frédéric Barbier, Marie-Elisabeth Ducreux, Matthias Middell, István Monok, Éva Ringh, Martin Svatoš

Volume VIII

École pratique des hautes études, Paris

École des hautes études en sciences sociales, Paris

Centre des hautes études, Leipzig

Bibliothèque nationale Széchényi, Budapest

Bibliothèque et centre d'information de l'Académie hongroise des sciences, Budapest 
LeS BIBLIOTHÈQUeS ET L'ÉCONOMIE DES CONNAISSANCES BibliotheKen und die ÖKonomie des Wissens

$$
145^{\circ}-185^{\circ}
$$

Colloque international - Internationale Tagung 9-13 avril/April 2019 Sárospatak (Hongrie/Ungarn)

Édité par

Frédéric Barbier, István Monok et Andrea Seidler

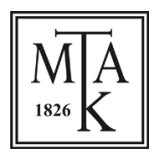

Magyar Tudományos Akadémia Könyvtár és Információs Központ Budapest 2020 
Mise en page

Viktória Vas

ISBN 978-963-7451-57-7

DOI 10.36820/SAROSPATAK.2020 
Préface

István MoNOK

Bibliothecae mutantur - Quare, quemadmodum et quid attinet?

Transformations de la composition thématique

des bibliothèques du Royaume de Hongrie aux $\mathrm{XV}^{\mathrm{E}}-\mathrm{XVI} \mathrm{I}^{\mathrm{e}}$ siècles....11

Marianne CARbonnier-Burkard

Les bibliothèques des Églises réformées françaises au XVII siècle.... 30

Max Engammare

De la bibliothèque de l'Académie de Calvin (1570) a la bibliothèque de l'Académie de Bèze (1612) à travers leur catalogue: Continuités et ruptures jusqu'au troisième catalogue de 1620........... 57

Róbert OLÁ́

Obsolescent Reformed Libraries in the seventeenth and eighteenth Century Carpathian Basin

Ádám Hegri

Moderner Zeitgeist - veraltete Lesestoffe. Bibliotheken reformierten Pfarrer um die Wende des 18. zum

19. Jahrhunderts im Königreich Ungarn

Petr MAšEK

Zierotin Library in Velké Losiny in Sixteenth and Seventeenth century. 136

Detlef HABERLAND

Schlesische Bibliotheken Zeichen der intellektuellen Vielfalt einer zentralen Bildungsregion in Europa 146

Thomas WaLLNIG

Sebastian Tengnagel und Johann Seyfried - Österreichische

Geschichtsschreibung zwischen Späthumanismus und

Gegenreformation 
Elisabeth EngL-Ursula RautenBerg

Christoph Jacob Trew - Bibliothek und Sammeln in der Gelehrtengemeinschaft der ersten Hälfte des 18. Jahrhunderts.

Helwi BLOM

Philosophie ou Commerce?

L'évolution des systèmes de classement bibliographique dans les catalogues de bibliothèques privées publies en France au XVIII ${ }^{\mathrm{e}}$ siècle.

Maria Luisa López-VIDrIero Abelló

Les meubles de la connaissance: façons de devenir sage à prix fixe.

Frédéric BARbier

Distinction, récréation, identité: la trajectoire des « romans» en France sous d'Ancien Régime.

\section{Andrea SeIdler}

Die praktische Bedeutung ungarischer Sammlungen und Bibliotheken für führende Gelehrte des Königreichs Ungarn im späten 18. Jahrhundert am Beispiel des Jesuiten Georg Pray (1723-1801).

\section{Olga Granasztói}

Se divertir: les enseignements de la bibliothèque d'une femme aristocrate hongroise à la fin du XVIII siècle

Christophe Didier

La naissance du théâtre „des boulevards”, ou Comment la banlieue entre en bibliothèque (1780-1830).

\section{Andrea De Pasquale}

La nascita delle riserve di libri antichi in Italia

Index des noms de personne et de lieu..... 